\title{
Neuromyotonia: an unusual presentation of intrathoracic malignancy
}

\author{
J. C. WALSH \\ From the Department of Neurology, Royal Prince Alfred Hospital, Sydney, NSW, Australia
}

SYNOPSIS A 48 year old woman is described who presented with increasing muscular rigidity and who was found to have a mediastinal tumour. Electrophysiological studies revealed that the muscular stiffness resulted from very high frequency motor unit activity which outlasted voluntary effort, and which was abolished by nerve block. The abnormal activity may have arisen at the anterior horn cell level. Marked improvement followed the administration of diphenylhydantoin.

There has been considerable interest in the unusual remote neurological manifestations of internal malignancy; cerebellar degeneration, transverse myelitis, peripheral neuropathy and myopathy, and the myasthenic syndrome are widely recognised complications. Abnormal muscular 'stiffness' is a very rare phenomenon and has been reported briefly in two patients with bronchogenic carcinoma (Humphrey et al., 1974; Waerness, 1974) and in one patient with a thymic tumour (Negri et al., 1974). Because of the rare nature of this condition, the following patient is described together with electrophysiological and histological studies.

\section{CASE REPORT}

A 51 year old woman (RPAH 365944) was admitted to another hospital in June 1974 for investigation of memory impairment, and of falling over for nine months. She had tripped and fallen for the first time in September 1973 at work and since then had increasing numbers of attacks of stumbling and falling; she also complained of some recent paraesthesiae in the arms and legs.

At the initial presentation she was alert, and well oriented but had poor recall for recent events; her gait was slow and shuffling but no other abnormality was demonstrated. An electroencephalogram, radiograph of the chest and skull, serum tests for syphilis, serum electrolytes, blood urea, haemoglobin, bloodfilm and ESR were all normal, and she was discharged from hospital. During the following two months she gradually 'slowed up' until June 1974 when she could

(Accepted 8 June 1976.) not get out of bed and was admitted to hospital. Her $\stackrel{0}{\circ}$ major symptom was an increasing number of falls, $\stackrel{\oplus}{\longrightarrow}$ usually occurring on rising from the sitting or lying $\overrightarrow{ }$ position; she would take a step, stiffen, totter, andoํㅇ fall. Her husband noted that on helping her to her feet the limbs were rigid but that after rising she could $\overrightarrow{0}$ usually shuffle along with very short steps. She also- $z$ complained of some tingling in the hands and feet. She had a cough, smoked heavily and had lost $20 \mathrm{~kg}$ weight during the six months before admission.

On examination she was now disoriented for botho time and place and her recent memory was very poor; she could not remember the names of three simple objects for a period of two minutes. There were no spontaneous facial or other movements; rapid shallow grunting respirations were noted and the speech was slow and nasal. There was slight left ptosis and very little tongue movement. The muscles were normal to inspection and to palpation, power was good but voluntary contraction appeared to persist after volition ceased and she walked in a slow shuffle with the legs rigidly extended. The reflexes were normal in the upper limbs, absent in the lower limbs and the plantar responses were flexor. Two point discrimination was $5 \mathrm{~mm}$ on the finger tips but appreciation of pinprick was reduced over the feet and hands.

The haemoglobin, white cell count, erythrocyte sedimentation rate, blood urea, serum magnesium, calcium, sodium, potassium, chloride, bicarbonate, bilirubin, uric acid, cholesterol, creatine phosphokinase, aspartate transaminase, thyroid function studies, and electrocardiogram were all normal; mild hyperglycaemia occurred during the glucose tolerance test. Radiological examination of the chest revealed a large mass lesion at the left hilum and within the 086 
anterior mediastinum together with enlargement of the hilar nodes (Fig. 1). Sputum cytology and bronchoscopy were normal but arterial hypoxaemia was present $\left(\mathrm{PaO}_{2} 59 \mathrm{mmHg}\right)$.

An electroencephalogram performed on admission contained some 4-7 $\mathrm{Hz}$ theta activity in the left temporal area. Two brain scans were normal and at pneumoencephalography there was evidence of bilateral cerebral atrophy. The spinal fluid was normal. A biopsy was obtained from the left triceps muscle. Electromyography was abnormal (see Results), and after these studies she was treated with diphenylhydantoin $100 \mathrm{mg}$ three times per day. Within four days there had been marked improvement in muscle stiffness and within two weeks she could walk almost normally, fully care for herself, sew and knit, and her speech and respiratory movements had returned to normal. Her mental deterioration progressed and she was transferred to a nursing home. The patient died four months after discharge. Permission for necropsy was refused.

\section{METHODS}

\section{ELECTROMYOGRAPHY}

Muscles were sampled with a concentric needle electrode (Disa 9013L 0311) and the signals led off to a Tektronix FM 122 preamplifier, which was $3 \mathrm{~dB}$ down at $8 \mathrm{~Hz}$ and $10 \mathrm{kHz}$, and displayed on one

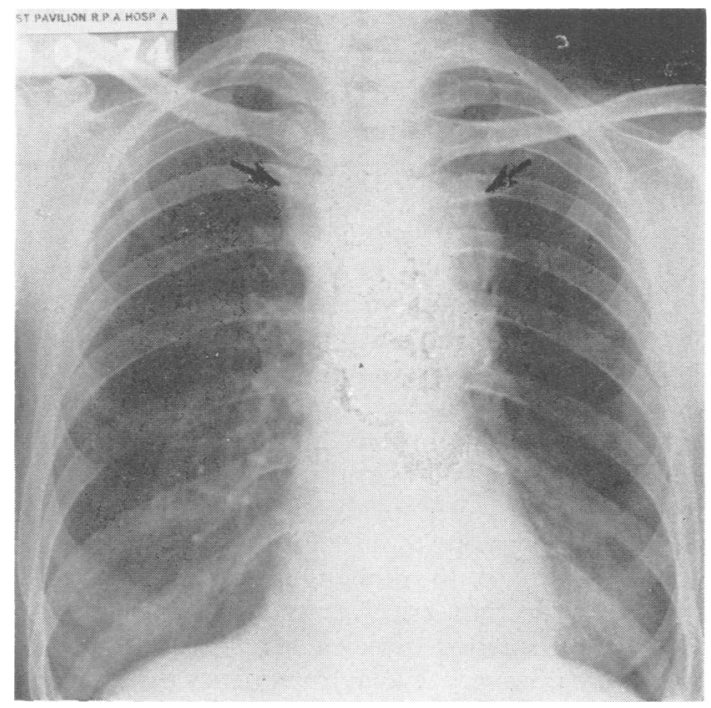

FIG. 1 Chest radiograph illustrating the mediastinal mass and enlargement of hilar nodes (arrows). channel of a Medelec M-Scope. A time scale derived from a Devices Digitimer, and a calibration signal derived from a Devices calibrate unit were displayed simultaneously on other channels. Nerve conduction studies were performed by methods standard in this laboratory (Walsh and McLeod, 1970). Electrical stimuli were derived from a Disa Ministim and applied via ring electrodes to the second and fifth fingers for evoking sensory action potentials in the median and ulnar nerves, and via surface electrodes to the median, ulnar, lateral popliteal, and sural nerves. Responses were recorded by surface electrodes except for responses obtained from the sural nerve for which subcutaneous needle electrodes were inserted alongside the nerve at the mid-calf level (Walsh, 1971).

\section{MUSCLE BIOPSY}

A biopsy was obtained from the left lateral head of triceps under local anaesthesia. A portion of the specimen was frozen and prepared for light microscopy and another portion was prepared for electron microscopy by methods standard in this laboratory (McLeod et al., 1975).

\section{RESULTS}

\section{ELECTROMYOGRAPHY}

The right lateral head of triceps, abductor pollicis brevis, abductor digiti minimi, and extensor digitorum brevis muscles were sampled with a concentric needle electrode. There was normal insertion activity and the muscles were silent at rest. On voluntary effort a normal interference pattern developed for several milliseconds but was then obscured by the sudden appearance of numerous motor unit potentials firing regularly at very high rates ranging from 100 300/s (Fig. 2C); the firing of individual motor units both started and ceased abruptly (Fig. 2A) and this activity was responsible for prolonged muscular contraction which outlasted the cessation of voluntary effort by up to 15 seconds. As the firing of the last high-frequency discharge in the territory of the recording electrode ceased there was usually electrical silence, although occasionally several fasciculating motor units, each with widely varying interdischarge intervals, would fire for up to two minutes. There was no spontaneous fibrillation or positive sharp wave activity detected. Occasionally, brief high frequency motor unit discharges were precipitated by manipulating the needle, but could not be produced by percussing the muscle. Similar activity was present almost continuously in the intercostal muscles (Fig. 2B).

The right ulnar nerve was blocked at the elbow by the local injection of $1 \%$ lignocaine. Electrical activity was studied in the abductor digiti minimi muscle with 

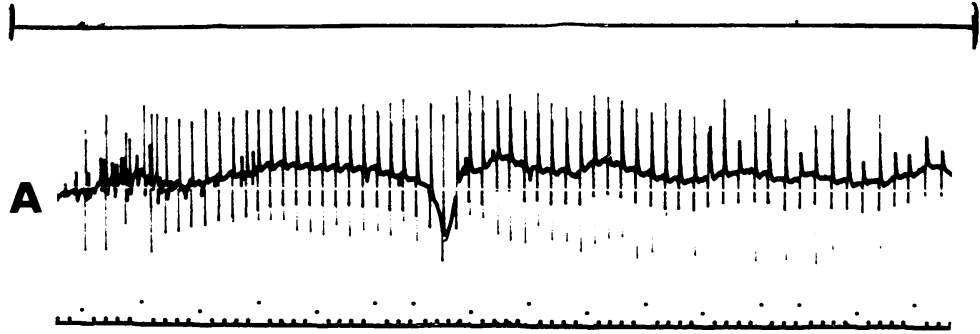

FIG. 2 (A) EMG activity in the right tibialis anterior muscle illustrating some of the last rapidly firing motor units to 'switch off' as involuntary

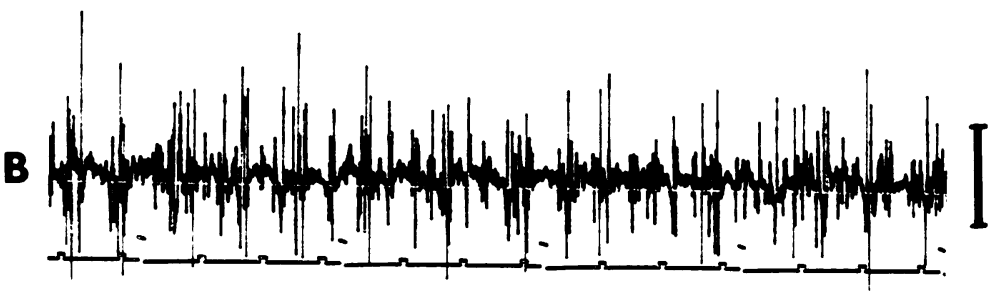
activity ceases several seconds after a voluntary contraction of the muscle. The larger unit is firing at 100/s. Scale: $1 \mathrm{~ms} /$ division. (B) Rapid bursts of EMG activity recorded from an external intercostal muscle. Scale: $10 \mathrm{~ms} /$ division. (C) Large

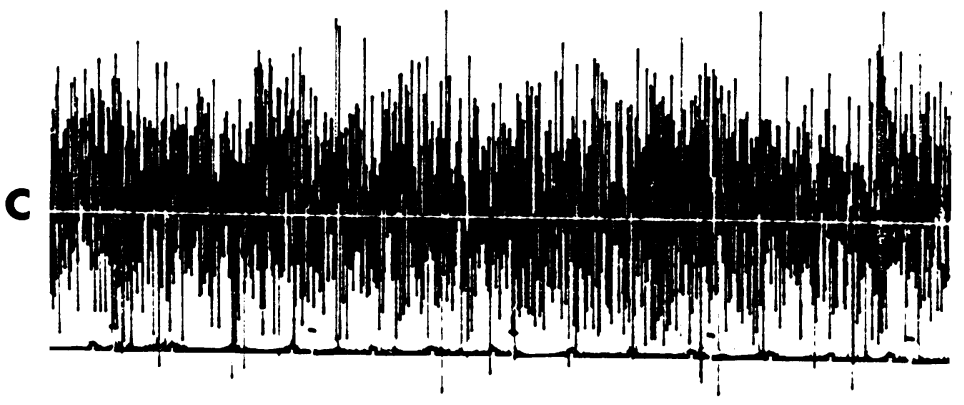
numbers of rapidly firing motor units in the abductor digiti minimi muscle during voluntary contraction. Scale: $10 \mathrm{~ms} /$ division. Vertical bar: $1 \mathrm{mV}$.

a concentric needle electrode, and the amplitude of the muscle action potential obtained in response to nerve stimulation was recorded via surface electrodes. Ten millilitres of lignocaine were infiltrated above the medial epicondyle and after 20 minutes there was almost complete paralysis of the ulnar-supplied muscles. A further $8 \mathrm{ml}$ was injected and after another 15 minutes there was no response to voluntary effort. Before injection, supramaximal electrical stimulation of the ulnar nerve $5 \mathrm{~cm}$ distal to the medial epicondyle produced an evoked muscle action potential followed by large numbers of high-frequency discharges lasting for up to 10 seconds. As paralysis developed, the amplitude of the evoked muscle action potential remained constant but the repetitive discharges were rapidly reduced in number until only one unit fired repetitively near the recording electrode (Fig. 3A, B). The latency from application of stimulus to the onset of abnormal motor unit activity was $27 \mathrm{~ms}$. When complete paralysis was obtained there was no activity recorded in response to stimulation above the elbow; and no repetitive firing occurred on stimulating below the level of the block, although the amplitude of the evoked muscle action potential remained unchanged (Fig. 3C).

Motor conduction velocities in the right median, ulnar and lateral popliteal nerves were $53 \mathrm{~m} / \mathrm{s}, 49 \mathrm{~m} / \mathrm{s}$, and $45 \mathrm{~m} / \mathrm{s}$ with terminal latencies of $3.5 \mathrm{~ms}, 2.3 \mathrm{~ms}$, and $5.7 \mathrm{~ms}$ respectively. Sensory action potentials of latency to peak $3.7 \mathrm{~ms}$ and $3.8 \mathrm{~ms}$ were recorded from the right median and sural nerves respectively, and the amplitude of the responses were $10 \mu \mathrm{V}$ and $7 \mu \mathrm{V}$; no response was recorded from the right ulnar nerve, and an H-reflex could not be elicited from the right calf muscles on stimulating the medial popliteal nerve.

Electromyography was repeated when a good clinical response to diphenylhydantoin had been obtained. The right triceps, abductor digiti minimi, and tibialis anterior muscles were re-examined and there were now very few high frequency discharges to be seen on effort; the involuntary activity that was present did not persist after the cessation of voluntary effort. 

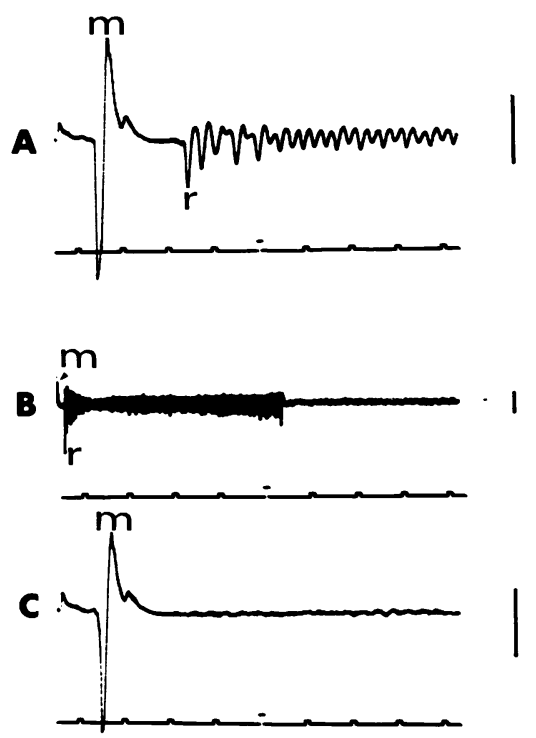

FIG. 3 Response recorded from the abductor digiti minimi muscle with a concentric needle electrode in response to stimulation of the ulnar nerve below the elbow with a partial $(A, B)$ and complete $(C)$ nerve block above the elbow. $M=$ direct response, $r=$ repetitive discharge. Scale $A, C=10 \mathrm{~ms} /$ division. $B=100$ ms/division. Vertical bar $1 \mathrm{mV}$.

\section{MUSCLE HISTOLOGY}

There was no cellular infiltration and the range of muscle fibre size was normal. An occasional fibre contained large lipid droplets (Fig. 4) and although most of the fibres were type 2 in the ATP-ase stain there were very few fibres in the sections that stained for glycogen with the PAS reaction. Electron microscopy confirmed the presence of abnormal collections of lipid in some fibres but no other abnormality was demonstrated.

\section{DISCUSSION}

There have been two previous reports of unusual muscular stiffness associated with bronchial carcinoma (Humphrey et al., 1974; Waerness, 1974), and a report of a patient with 'myotonia' related to a thymic tumour (Negri et al., 1974), although detailed electrophysiological studies have not been published.

This patient presented with incapacitating muscular stiffness which was precipitated by movement

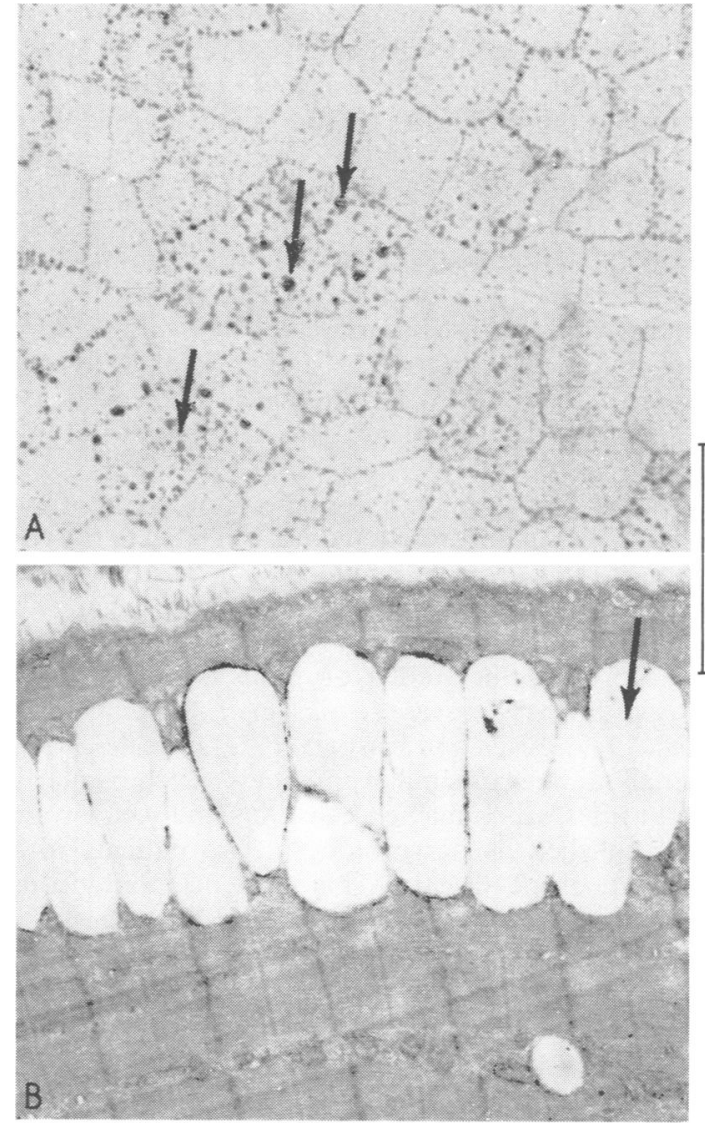

FIG. 4 Light $(A)$ and electron micrographs $(B)$ illustrating increased numbers of lipid droplets within some fibres (arrows). A: scale $200 \mu \mathrm{m}$; Oil Red O. B: scale $2 \mu \mathrm{m}$; Toluidine blue.

and which outlasted the cessation of voluntary effort by up to 30 seconds. The electromyographic studies confirmed that the muscles were electrically silent at rest, and that large numbers of bizarre, high-frequency motor unit discharges appeared during voluntary effort. These findings may be compared with those obtained in other disorders associated with muscular stiffness. The electromyographic abnormalities were not those of myotonia, and were completely abolished by nerve block; this observation indicates that the abnormality was present at a pre-junctional site and thus the term 'neuromyotonia' could be applied to the disorder. There is some clinical similarity between this patient and patients with the 'stiff-man syndrome' (Moersch and Woltman, 1956) but electromyography 
has demonstrated continuous but normal motor unit activity in this group of patients (Trethowan et al., 1960; Howard, 1963; Kasperek and Zebrowski, 1971). Similarly, the syndrome of continuous muscle fibre activity (Isaacs, 1961) is associated with relatively normal motor unit activity, together with fasciculations in some cases (Wallis et al., 1970; Isaacs and Frere, 1974) and with doublet and triplet motor unit discharges in other cases (Wadia and Irani, 1975). Finally, although intense muscular rigidity may arise after functional 'isolation' of the anterior horn cell, this is associated with continuous anterior horn cell discharge at rates of up to 30/s (Rushworth et al., 1961).

There is some evidence to suggest however that the abnormality in the present patient is related to the anterior horn cell. Small numbers of bizarre, highfrequency motor unit discharges, which begin and cease abruptly, are occasionally demonstrated during electromyographic studies on patients with chronic anterior horn cell disease (Amick et al., 1966; Munsat et al., 1969). Eisen and Karpati (1971) have described a small number of similar potentials which regularly fire at rates which may exceed $100 / \mathrm{s}$ and which were not abolished by nerve block in two patients with motor neurone disease. This type of spontaneous activity appears to be asymptomatic because of the small numbers of motor units affected. By contrast, large numbers of discharges occurred in the muscles of this patient during effort, and although needle movement would produce an occasional brief single train of discharges the muscles were silent at rest. Discharges related to needle movement do not necessarily indicate local hyperexcitability as the pain of needle movement could produce brief reflex activation of some motor units. The effect of complete nerve block clearly indicates the pre-junctional site of the abnormality, and the response recorded from the abductor digiti minimi muscle on stimulating the ulnar nerve at the elbow below the level of an almost complete block suggests that the repetitive activity may arise at the level of the anterior horn cell, as the interval between the direct evoked muscle action potential and the onset of firing of a high-frequency discharge was of the order of $25 \mathrm{~ms}$. This interval would be sufficient time for an antidromic impulse in the motor fibres to reach the anterior horn cell and for a repetitive discharge to return to the muscle. The muscle stiffness in the patient reported by Humphrey et al. (1974) subsided spontaneously in spite of the progression of the malignancy but the response to diphenylhydantoin was both rapid and dramatic in this patient and was almost certainly significant. For these reasons it is suggested that in the present patient there was disinhibition of anterior horn cells resulting in rapid repetitive discharges in response to a single depolar- ization, and that diphenylhydantoin was effective because this drug stabilises the nerve cell membrane.

The pathological nature of the mediastinal tumour could not be established, although the radiological appearances, recent weight loss, cough, and heavy tobacco consumption together suggest that the diagnosis was bronchogenic carcinoma. In spite of this uncertainty the unusual nature of the electrophysiological abnormalities merits attention so that detailed investigations may be performed on similar cases. It was of interest that most fibres in the muscle biopsy were depleted of glycogen, and that some fibres contained increased amounts of lipid. These changes are probably secondary to an unusually high level of muscle activity.

The expert technical assistance of Miss Julia Baverstock is gratefully acknowledged.

\section{REFERENCES}

Amick, L. D., Smith, H. L., and Johnson, W. W. (1966). An unusual spectrum of progressive spinal muscular atrophy. Acta Neurologica Scandinavica, 42, 275-295.

Eisen, A. A., and Karpati, G. (1971). Spontaneous electrical activity in muscle. Journal of the Neurological Sciences, 12, 212-235.

Howard, F. M. (1963). A new and effective drug in the treatment of the stiff man syndrome. Mayo Clinic Pro ceedings, 38, 203-212.

Humphrey, J. G., Hill, M. E., Gordon, A. S., and Kalow W. (1974). Myotonia associated with small cell carcinoma of lung. In 3rd International Congress on Muscle Diseases, Newcastle-upon-Tyne. (Abstract 355.) Excerpta Medica: Amsterdam.

Isaacs, H. (1961). A syndrome of continuous muscle-fibre activity. Journal of Neurology, Neurosurgery, and Psychiatry, 24, 319-325.

Isaacs, H., and Frere, G. (1974). Syndrome of continuous muscle fibre activity. South African Medical Journal, 10, 1601-1007.

Kasperek, S., and Zebrowski, S. (1971). Stiff man syndrome and encephalomyelitis. Archives of Neurology (Chic.), 24, 22-30.

McLeod, J. G., Baker, W. de C., Shorey, C. D., and Kerr, C. B. (1975). Mitochondrial myopathy with multisystem abnormalities and normal ocular movements. Journal of the Neurological Sciences, 24, 39-52.

Moersch, F. P., and Woltman, H. W. (1956). Progressive fluctuating muscular rigidity and spasm. Mayo Clinic Proceedings, 31, 421-426.

Munsat, T., Woods, R., Fowler, W., and Pearson, C. M. (1969). Neurogenic muscular atrophy of infancy with prolonged survival. Brain, 92, 9-24.

Negri, S., Boiardi, A., and Bussone, G. (1974). Transitory myotonia after thymectomy. In 3rd International Congress on Muscle Diseases, Newcastle-upon-Tyne. (Abstract 357.) Excerpta Medica: Amsterdam. 
Rushworth, G., Lishman, W. A., Hughes, J. T., and Oppenheimer, D. R. (1961). Intense rigidity of the arms due to isolation of motoneurones by a spinal tumour. Journal of Neurology, Neurosurgery, and Psychiatry, 24, 132-142.

Trethowan, W. H., Allsop, J. L., and Turner, B. (1960). The 'stiff man' syndrome. Archives of Neurology (Chic.), $3,448-456$.

Wadia, N. H., and Irani, P. F. (1975). Continuous muscle fibre activity (Isaac syndrome)-evidence to suggest a proximal neurogenic causation. Proceedings of the 4th Asian and Oceanian Congress of Neurology, Bangkok, Thailand, p. 87.
Waerness, E. (1974). Neuromyotonia and bronchial carcinoma. In 3rd International Congress on Muscle Diseases, Newcastle-upon-Tyne, p. 152. (Abstract 356.) Excerpta Medica: Amsterdam.

Wallis, W. E., van Poznak, A., and Plum, F. (1970). Generalised muscular stiffness, fasciculations and myokymia of peripheral nerve origin. Archives of Neurology (Chic.), 22, 430-439.

Walsh, J. C. (1971). The neuropathy of multiple myeloma. Archives of Neurology (Chic.), 25, 404-414.

Walsh, J. C., and McLeod, J. G. (1970). Alcoholic neuropathy. An electrophysiological and histological study. Journal of the Neurological Sciences, 10, 457-469. 